\title{
THOMSON SCATTERING IN MAGNETIC FIELDS
}

\author{
Barbara Whitney \\ Department of Astronomy \\ University of Wisconsin-Madison
}

\begin{abstract}
The equation of transfer in Thomson scattering atmospheres with magnetic fields is solved using Monte Carlo methods. Two cases, a plane parallel atmosphere with a magnetic field perpendicular to the atmosphere, and a dipole star, are investigated. The wavelength dependence of polarization from a plane-parallel atmosphere is qualitatively similar to that observed in the magnetic white dwarf Grw $+70^{\circ} 8247$, and the field strength determined by the calculation, $3.2 \times 10^{8} \mathrm{G}$, is quantitatively similar to that determined from the line spectrum. The dipole model does not resemble the data as well as the single plane-parallel atmosphere.
\end{abstract}

\section{INTRODUCTION}

The most strongly magnetic white dwarfs have surface field strengths in the range of $10^{8}-10^{9} \mathrm{G}$. In order to explain the strong linear and circular polarization of these objects, one needs to know the polarization and angular dependence of the important absorption processes in a magnetic white dwarf. Several people (e.g., Martin and Wickramasinghe 1978, Wickramasinghe and Martin 1979, Wickramasinghe and Ferrario 1988, Nagendra and Peraiah 1984) have modelled the continuum polarization spectra of magnetic white dwarfs using the dichroic opacities of Kemp (1977) for free-free absorption, and Lamb and Sutherland (1974) for bound-free. Both of these opacities were derived in the weak field limit, $\omega_{\mathrm{c}} / \omega \ll 1\left(\omega_{\mathrm{c}}=\mathrm{eB} / \mathrm{mc}\right.$, the cyclotron frequency). For large field strength, that is, $\omega_{c} / \omega \gg 1$, the motion of electrons is confined along the field lines, and the opacity expressions of Lodenquai et al. (1974) can be used, though they do not provide a complete description of the angular dependence of the cross sections. Lauer et al. (1983) present polarization and angular dependence of bremsstrahlung for the case of $h \omega \ll k T \ll h \omega_{c}$ in fields so strong that electron motion perpendicular to the field is confined to the first Landau level. For weaker fields $\left(\omega<\omega_{c}\right)$ and $h \omega<k T$, Stokes parameters for free-free absorption are given by Wickramasinghe and Meggitt (1985).

For $k T \sim h \omega \sim h \omega_{c}$, as is the case for the atmospheres of the strongest magnetic white dwarfs, a continuum opacity for which the polarization and angular dependence of the cross sections is known exactly is Thomson scattering. Since the behavior of other opacities may be similar to that of Thomson scattering, it is instructive to examine the polarization properties of a Thomson scattering atmosphere. 


\section{MODEL ATMOSPHERES}

Stokes parameters for electron scattering in a constant magnetic field along $\mathrm{z}$ were derived classically using the method of Chou (1986), and then integrated to give cross sections which depend on direction and polarization of the incident radiation. These cross sections agree with Herold (1979) in the non-relativistic limit. Radiative transfer in a plane parallel atmosphere with magnetic field along $\mathrm{z}$ was solved using a Monte Carlo method. For arbitrary field orientations, the radiative transfer was solved in an atmosphere that was tilted with respect to the $\mathrm{z}$-axis, and the results transformed into the coordinate frame of the atmosphere.

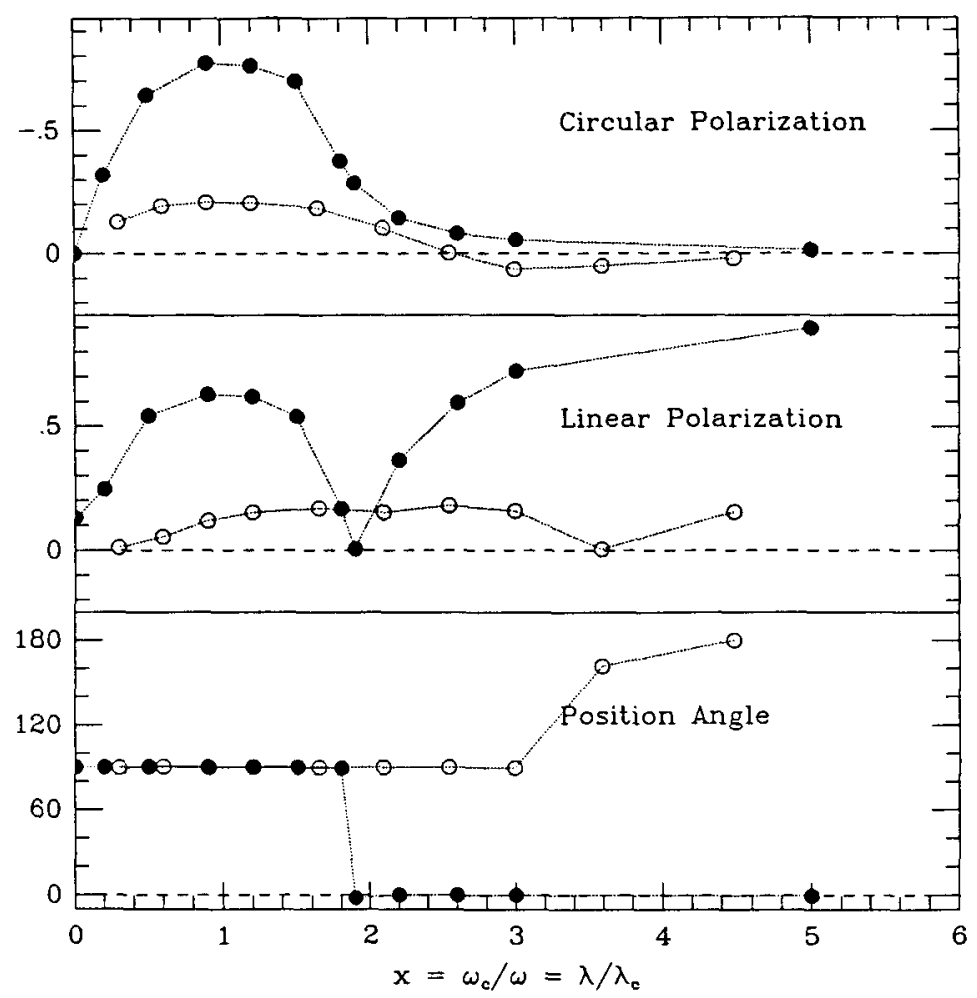

Figure 1 - Fractional polarization $(1=100 \%)$ is plotted as a function of $\mathrm{x}=\omega_{\mathrm{c}} / \omega=$ $\lambda \lambda_{c}$. The filled circles show polarization from a plane-parallel atmosphere with magnetic field along the $-\mathrm{z}$ axis, perpendicular to the atmosphere. The viewing angle is $60^{\circ}$ from the $\mathrm{z}$-axis. Open circles are from a dipole model viewed $60^{\circ}$ from the south pole.

The filled circles of Figure 1 show the results of a model atmosphere with the magnetic field along the negative $z$ axis, and an optical depth of three. Unpolarized grey light is incident normal to the bottom of the atmosphere. The polarization of scattered light emerging from the top at an angle of $60^{\circ}$ 
from the normal is shown here for several different values of $x=\omega_{c} / \omega=\lambda / \lambda_{c}$. At this angle the linear polarization is comparable to the circular, as it is in $\mathrm{Grw}+70^{\circ} 8247$. As expected, the circular polarization is largest at the cyclotron frequency, that is, $\omega=\omega_{c}$. An interesting feature of the linear polarization is the change in position angle at $\omega_{\mathrm{c}} / \omega=1.9$. This jump occurs at all exit angles. It can be understood as follows: When no field is present, radiation emerging from a plane parallel semiinfinite Thomson scattering atmosphere is linearly polarized with a position angle of $90^{\circ}$ with respect to the zenith (Chandrasekhar, 1960). In weak magnetic fields, the cross section for scattering is large when the electric vector of the incoming photon is perpendicular to the field, which in this case is also perpendicular to the atmosphere. This gives rise to linear polarization with a position angle of $90^{\circ}$. When the cyclotron frequency becomes much greater than that of the incident radiation, the electron can only be excited at the frequency $\omega$ when the electric vector of the incident radiation is parallel to the field. The scattered radiation is linearly polarized at a position angle of $0^{\circ}$. With this behavior in mind, we can interpret the results of a dipole model.

\section{A DIPOLE MODEL}

A grid of plane-parallel atmospheres was solved, each atmosphere having a magnetic field orientation and strength appropriate for its latitude on the surface of a dipole sphere. The outgoing radiation was integrated over the surface of the sphere. The optical depth of each atmosphere is $\tau=3$, because it takes less computing time than larger depths and it gives similar answers. The difference in polarization between an atmosphere of $\tau=3$ and $\tau=6$ depends on field strength, but is never more than $20 \%$ for $\omega_{\mathrm{c}} / \omega$ greater than 0.1 .

Since Monte Carlo solutions provide outgoing radiation at all angles, the dipole star can be rotated and viewed at any angle. The open circles in Figure 1 show the results for a viewing angle of $60^{\circ}$ from the south pole. Again, $60^{\circ}$ was chosen because the linear polarization is comparable to circular at this angle. The results are different than those in the plane-parallel case. The circular polarization changes sign at $\omega_{\mathrm{c}} / \omega \approx 3$ even though we are looking down the south pole and so expect only negative polarization. The reason for this is that the negative polarization from the south pole is small at $\omega_{\mathrm{c}} / \omega \approx 3$ but the positive polarization from the equator, where the field lines are pointing away from us, is large because there $\omega_{\mathcal{c}} / \omega \approx 1.5$. Another difference from the plane-parallel atmosphere is that the position angle of the linear polarization does not rotate until $\omega_{\mathrm{c}} / \omega \approx 3.5$. Both the linear and circular polarization are not as large as in the single atmosphere.

\section{COMPARISON WITH OBSERVATIONS}

Continuum polarization data of $\mathrm{Grw}+70^{\circ} 8247$ have been obtained by Landstreet and Angel (1975). The magnitude of the circular polarization has a narrow absorption feature at $3400 \mathrm{~A}$, increases to $-5 \%$ at $4500 \mathrm{~A}$, and then decreases with increasing wavelength. The linear polarization peaks at $4 \%$ at about $3900 \mathrm{~A}$, then decreases to less than $1 \%$ by $5000 \mathrm{~A}$. Its position angle rotates by $90^{\circ}$ at about $5700 \mathrm{~A}$. The polarization then starts to increase at about $7500 \mathrm{~A}$ and reaches $6 \%$ at $10000 \mathrm{~A}$. 
If we match the wavelength of the position angle rotation of the single atmosphere model shown in Figure 1 (solid dots) to the rotation of the data at $5700 \mathrm{~A}$, then the cyclotron wavelength of the atmosphere is $\lambda_{c}=\lambda / \mathrm{x}=5700 / 1.9=3353 \mathrm{~A}$, which corresponds to a field strength of $3.2 \times 10^{8} \mathrm{G}$. Angel, Liebert, and Stockman (1985) estimated the field strength of Grw $+70^{\circ} 8247$ to be $(1.6-3.5) \times 10^{8} \mathrm{G}$ from identification of hydrogen lines. Henry and $\mathrm{O}^{\prime}$ Connell (1984) estimated the field to be (4.0-7.6) $\times 10^{8} \mathrm{G}$ from the shifted wavelength of Ly- $\alpha$. The similarity of the polarization and field strength of the plane parallel atmosphere to $\mathrm{Grw}+70^{\circ} 8247$ is intriguing. However, the plane parallel model does not exactly fit the data: The dip in linear polarization at $5700 \mathrm{~A}$ is much wider in the data than in the model, and the circular polarization of the data decreases more slowly with wavelength than the model.

The dipole model does not fit the data very well. The field strength required to match the rotation of the position angle is $6.7 \times 10^{8} \mathrm{G}$ at the pole (half that at the equator). This would put the peak of the circular polarization at $1600 \mathrm{~A}$. The circular polarization would change sign at $4100 \mathrm{~A}$, which is not at all like the data. If we match the circular polarization peak to the data, then of course the problem is that the position angle rotation occurs at about twice the wavelength of the data.

The dipole model can be modified so that more radiation is incident on the atmospheres at the poles than at the equator. This brings the wavelength of the position angle rotation down with respect to the circular polarization peak, and in fact widens out the linear polarization dip to look more like the data. But to get this fit requires 25 times more radiation at the poles than at the equator, which may be unreasonable.

This work is supported by contract NAS5-26777 for the Wisconsin Ultraviolet Photo-Polarimeter Experiment.

\section{REFERENCES}

Angel, J. R. P., Liebert, J., and Stockman, H. S. 1985, Ap. J., 292, 260.

Chandrasekhar, S. 1960, Radiative Transfer (New York: Dover).

Chou, C. K. 1986, Astrophys. and Space Sci., 121, 333.

Henry, R. J. W., and O'Connell, R. F. 1984, Ap. J., 282, L97.

Herold, H. 1979, Phys. Rev. D, 19, 2868.

Kemp. J. C. 1977, Ap. J., 213, 794.

Lamb, F. K., and Sutherland, P. G. 1974, in IAU Symposium 53, Physics of Dense Matter, ed. C. J. Hansen (Dordrecht: Reidel), p. 265.

Landstreet, J. D., and Angel, J. R. P. 1975, Ap. J., 196, 819.

Lauer, J., Herold, H., Ruder, H. and Wunner, G. 1983, J. Phys.B: At. Mol Phys., 16, 3673.

Lodenquai, J., Canuto, V., Ruderman, M., Tsuruta, S. 1974, Ap. J., 190, 141.

Martin, B., and Wickramasinghe, D. T. 1978, Mon. Not. R. astr. Soc, 183, 533

Nagendra, K. N., and Peraiah, A. 1984, Astrophys. and Space Sci., 104, 61.

Wickramasinghe, D. T., and Ferrario, L. 1988, Ap. J., 327, 222.

Wickramasinghe, D. T., and Martin, B. 1979, Mon. Not. R. astr. Soc., 188, 165.

Wickramasinghe, D. T., and Meggitt, S. M. A. 1985, Mon. Not. R. astr. Soc., 214, 605. 